\title{
Tumor venéreo transmisible diseminado sobre piel, párpados y pene en un perro. Reporte de caso
}

\author{
Disseminated transmissible venereal tumor on skin, eyelids and genital in a male dog. Case report
}

\author{
J Ojeda ${ }^{a *}$, A Alfaro ${ }^{\mathrm{a}}$, M Moroni ${ }^{\mathrm{b}}, \mathrm{V}$ Camacho $^{\mathrm{c}}, \mathrm{J}_{\text {Martínez }}^{\mathrm{c}}$, M Noro ${ }^{\mathrm{a}}$ \\ ${ }^{a}$ Instituto de Ciencias Clínicas Veterinarias, Facultad de Ciencias Veterinarias, Universidad Austral de Chile, Valdivia, Chile. \\ bInstituto de Patología Animal, Facultad de Ciencias Veterinarias, Universidad Austral de Chile, Valdivia, Chile. \\ ${ }^{c}$ Centro de Investigación y Estudios Avanzados en Salud Animal, Facultad de Medicina Veterinaria y Zootecnia, Universidad \\ Autónoma del Estado de México, México.
}

\begin{abstract}
SUMMARY
Canine venereal transmissible tumor (CTVT) is a round cell benign neoplasia that affects the external genital mucous which is transmitted during coitus with infected dogs. The normal canine behaviour such as smelling and sucking allow the tumoral cells to implant into extragenital zones with subsequent metastases formation. This report described a dog with multiple skin nodules localised in the thoracolumbar area and eyes. The nodules were ulcered and alopecic with purulent fluid around them. A granulomatous mass with focal necrosis area was also detected with bilateral keratitis. Citological analysis of skin nodules, eyelid tissue and granulomatous tissue on the base of the penis suggested a CTVT, and the histopathological analysis confirmed the diagnosis of CTVT. Also LINE-1 rearrangement in c-myc was detected as a genetic alteration of the CTVT. A chemotherapy protocol was started with vincristine sulfate. Haemograms were used previous to each chemotherapy sessions to prevent myelosuppression. A moderate non regenerative anemia was observed after the third session but it was resolved by discontinuing chemotherapy for two weeks. The chemotherapy was effective to eliminate the CTVT after six sessions.
\end{abstract}

Key words: canine transmissible tumor, skin, vincristine, dogs.

\section{RESUMEN}

El tumor venéreo transmisible canino (TVT) es una neoplasia benigna de células redondas que afecta frecuentemente la mucosa genital externa de perros. El olfateo y el lamido permiten la implantación de las células tumorales en zonas extragenitales con formación de metástasis. Se relata un caso de TVT diseminado en piel, párpados y pene en un perro macho mestizo. Las lesiones localizadas en la parte dorsal y lateral de la zona torácica y abdominal presentaban varios nódulos alopécicos ulcerados y bien vascularizados, con aspecto friable y con secreción purulenta. El párpado superior izquierdo y derecho presentaban una masa granulosa con áreas de necrosis y queratitis bilateral. En la base del pene se presentaba una masa granulosa firme y bien delimitada. El análisis citológico de los nódulos cutáneos, de los párpados y la masa del pene reveló hallazgos compatibles con TVT, el que fue confirmado con análisis histopatológico. Además se determinó la presencia de LINE-1 en el oncogen c-myc como una alteración genética conocida para CTVT. Se estableció un protocolo quimioterápico sobre la base de sulfato de vincristina. Previo a cada sesión de quimioterapia se tomaron hemogramas para prevenir mielosupresión. Posterior a la tercera sesión se observó una anemia no regenerativa moderada que fue resuelta suspendiendo durante 2 semanas la terapia. La terapia con sulfato de vincristina fue efectiva para producir la regresión de todos los tumores en 6 sesiones, sin mostrar recurrencia.

Palabras clave: tumor venéreo transmisible, metástasis, piel, canino.

\section{INTRODUCCIÓN}

El tumor venéreo transmisible canino (TVT), granuloma venéreo, tumor de Sticker o sarcoma de Sticker, es una neoplasia benigna de células redondas que afecta frecuentemente la mucosa genital externa de perros machos y hembras. El TVT se transmite sexualmente, mediante transferencia de células neoplásicas exfoliadas intactas de un animal para otro. La implantación tumoral requiere una mucosa genital receptora previamente lesionada, como ocurre en el coito (Park y col 2006), constituyendo un

Aceptado: 06.08.2015.

* javierojeda@uach.cl aloinjerto natural (Ortega-Pacheco y col 2003). El TVT se caracteriza por ser una masa blanda solitaria o múltiple, multilobulada sangrante con forma de coliflor, friable, de color rosado, que puede estar ulcerada e infectada y, en muchos casos, con apariencia necrótica (Ortega-Pacheco y col 2003). La localización más frecuente en machos es desde la parte caudal del pene hasta el bulbo del glande, y en hembras en la conjunción de la vagina y el vestíbulo con descarga sanguinolenta o sangrado prepucial o vaginal; donde los signos asociados con el tumor pueden ser confundidos con el celo o con afecciones como cistitis, prostatitis o uretritis (Grandez y col 2011).

Otras formas de transmisión son el olfateo y el lamido que permiten la implantación de las células tumorales en zonas extragenitales como vía nasal y oral (Levy y col 2006), 


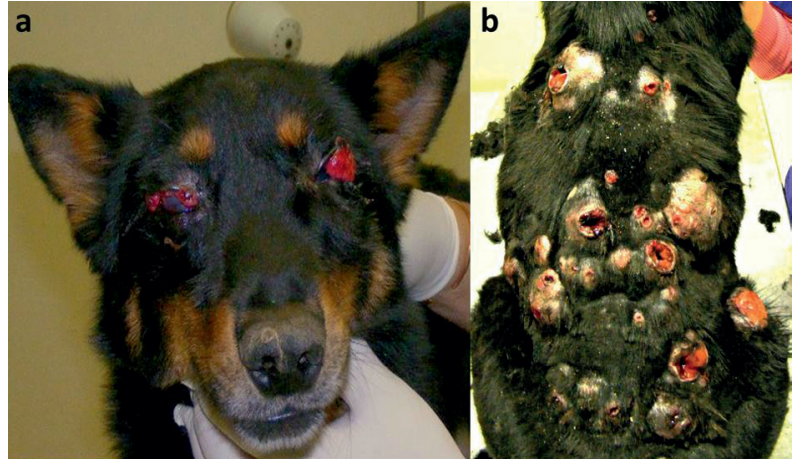

Figura 1. Localización y aspecto de las lesiones observadas sobre el perro. Tejido granulomatoso diseminado en zona parpebral bilateral con opacidad corneal (a). Nódulos ulcerados y con fluido purulento en zona toracolumbar (b).

Localization and aspect of the nodules observed on dog. Diseminated granulomatous tissue on eyelids with corneal opacity (a) Ulcerated nodules with purulent fluid on toracolumbar zone (b).

piel (Marcos y col 2006, Park y col 2006), ojo y conjuntiva (Ferreira y col 2000) y, región perianal y escroto (OrtegaPacheco y col 2003). Los tumores primarios normalmente se localizan en la mucosa de los genitales externos, aunque se han reportado casos de implantación extragenital primaria sin compromiso genital previo (Marcos y col 2006). Las metástasis localizadas en pulmones, vísceras abdominales (Park y col 2006) y en el sistema nervioso central son raras (Kangasniemi y col 2004, Ferreira y col 2000). El TVT presenta bajo potencial metastásico, es por eso que la presencia de células neoplásicas en tejidos distintos al genital es poco frecuente (Grandez y col 2011).

\section{MATERIAL Y MÉTODOS}

\section{HISTORIA Y HALLAZGOS CLÍNICOS}

Un macho adulto mestizo de aproximadamente 1 año de edad fue derivado con antecedentes de presentar nódulos en la piel hace 3 meses que comenzaron a tener secreción purulenta. Historial de vacunas y desparasitaciones no se conocía, ya que el perro fue recogido de la calle.

Al examen físico, el animal se encontraba decaído con lenta respuesta a los estímulos externos observándose una baja condición corporal (2/5). Las mucosas orales y oculares se encontraban levemente congestivas sin rasgos de deshidratación. La temperatura, frecuencia respiratoria y cardíaca se encontraban dentro del intervalo de normalidad. El pelaje se presentaba brillante, con buena implantación. La zona cutánea de la parte dorsal y lateral de la zona torácica y abdominal presentaba varios nódulos alopécicos ulcerados, bien vascularizados, con aspecto friable y secreción purulenta. Muchos de estos nódulos estaban cubiertos por pelaje (figura $1 b$ ). Se observó también una masa granulosa en el párpado superior del lado izquierdo y derecho con presencia de áreas de necrosis (figura 1a) y queratitis bilateral sin presentar signos asociados a ceguera. No se detectaron lesiones orales.

Como prediagnóstico se indicó hipersensibilidad a picaduras de insectos, piogranuloma o mastocitoma generalizado.

Se tomaron muestras sanguíneas para análisis hematológicos y bioquímicos. Además, se realizó punción con aguja fina e impronta de las lesiones en piel y párpados para examen citológico. También se realizó punción con aguja fina de los nódulos linfáticos mandibulares, preescapulares y poplíteos.

El análisis hematológico demostró la presencia de leucocitosis con neutrofila con desvío a la izquierda regenerativa y aumento relativo del hematocrito, ya que el número de eritrocitos se mantuvo dentro del rango (cuadro 1). Los parámetros bioquímicos analizados como ALT, GGT, fosfatasa alcalina, AST, urea, creatinina y albúmina se encontraban en rangos de normalidad (cuadro 1).

Las muestras de impronta obtenidas de las lesiones fueron coloreadas con tinción Giemsa. El análisis citológico de las masas ubicadas en el dorso reveló presencia de alta celularidad, constituida por células ovoides, con núcleo redondo, grande, principalmente excéntrico y basofílico y la presencia de nucléolos prominentes y numerosas figuras mitóticas. El citoplasma basofílico o transparente contenía múltiples vacuolas claras. Abundante presencia de neutrófilos y bacterias Gram negativas (figura 2). Similares hallazgos fueron encontrados en las lesiones ubicadas en los párpados. Los nódulos linfáticos presentaron gran cantidad de linfocitos de pequeño tamaño. Además se observó la presencia de numerosas células plasmáticas con varios grados de diferenciación caracterizadas por abundante citoplasma con presencia de vacuolas esféricas y blanquecinas. Escasa presencia de macrófagos y neutrófilos. Estos resultados fueron consistentes con TVT, tanto en piel y párpados. Por

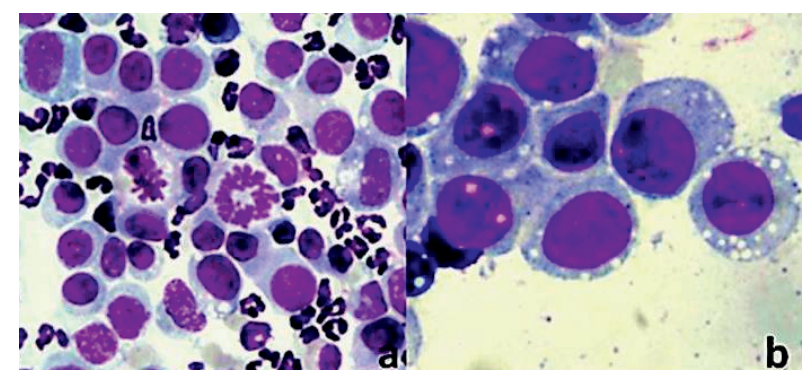

Figura 2. Examen citológico de impronta de tumor venéreo transmisible canino con nódulos cutáneos (a, 40x) y masa en pene (b, 100x). Se observa anisocariosis, núcleo grande, nucléolos prominentes, escaso citoplasma basófilo con vacuolas, figuras en mitosis y presencia de polimorfonucleares, especialmente en los nódulos cutáneos. Tinción Giemsa.

Citological exam of the CTVT with skin nodules $(\mathrm{a}, 400 \mathrm{x})$ and penis mass $(\mathrm{b}, 1000 \mathrm{x})$. It is possible to observe anisocariosis, prominent nucleus and nucleolus with lacking basophil cytoplasm. Multiple vacuoles and mitotical figures are observed. Abundant polymorphonuclear neutrophils are observed, especially in cutaneous nodules. Giemsa stain. 
Cuadro 1. Resultados de los exámenes hematológicos y bioquímicos del perro con TVT previo al tratamiento con vincristina. Hematology and serum biochemistry results from canine with CTVT before chemotherapy.

\begin{tabular}{lcc}
\hline Parámetro & Valores obtenidos & Rango de referencia \\
\hline Eritrocitos & 8, cels x $10^{6} \mu \mathrm{L}$ & $5,5-8,5$ cels x $10^{6} \mu \mathrm{L}$ \\
Hematocrito & $56 \%$ & $37-50 \%$ \\
Plaquetas & $300 \mathrm{cels} \times 10^{3} \mu \mathrm{l}$ & $300-500 \mathrm{cels} \times 10^{3} \mu \mathrm{l}$ \\
Leucocitos totales & $17.600 \mathrm{cels} / \mu \mathrm{L}$ & $8.000-14.000 \mathrm{cels} / \mu \mathrm{L}$ \\
Neutrófilos & $14.960 \mathrm{cels} / \mu \mathrm{L}$ & $3.300-10.000 \mathrm{cels} / \mu \mathrm{L}$ \\
Baciliformes & $600 \mathrm{cels} / \mu \mathrm{L}$ & $0-300 \mathrm{cels} / \mu \mathrm{L}$ \\
ALT & $35 \mathrm{U} / \mathrm{L}$ & $25-85 \mathrm{U} / \mathrm{L}$ \\
GGT & $8 \mathrm{U} / \mathrm{L}$ & $2-10 \mathrm{U} / \mathrm{L}$ \\
AST & $59 \mathrm{U} / \mathrm{L}$ & $30-90 \mathrm{U} / \mathrm{L}$ \\
Fosfatasa alcalina & $118 \mathrm{U} / \mathrm{L}$ & $40-132 \mathrm{U} / \mathrm{L}$ \\
Urea & $8,7 \mathrm{mmol} / \mathrm{L}$ & $2,6-6,6 \mathrm{mmol} / \mathrm{L}$ \\
Creatinina & $88 \mu \mathrm{mol} / \mathrm{l}$ & $35-15 \mu \mathrm{mol} / \mathrm{L}$ \\
Albúmina & $55 \mathrm{~g} / \mathrm{L}$ & $25-85 \mathrm{~g} / \mathrm{L}$ \\
\hline
\end{tabular}

otra parte, los nódulos linfáticos se encontraban bajo un proceso inflamatorio crónico. De acuerdo con las características celulares el TVT se subclasificó como plasmocítico.

Sobre la base de los hallazgos citológicos se realizó un examen de genitales, no realizado anteriormente por el evidente compromiso cutáneo. En la base del pene se observó una masa granulosa firme y bien delimitada (figura 3) con características citológicas similares a las masas de piel y párpados. El prepucio no presentaba lesiones.

Para confirmar el diagnóstico se tomaron biopsias de las masas observadas. Utilizando una tinción de hematoxilinaeosina se observó en todas las muestras una marcada y amplia proliferación celular muy uniforme y compacta, con múltiples fibras de tejido conectivo, dando un aspecto trabeculado al tejido. Las células presentaron una forma redonda a poliédrica con escaso citoplasma rosado pálido y núcleos ovoides de gran tamaño con abundante cromatina granular fina y un nucléolo eosinofílico muy evidente. Múltiples figuras mitóticas, además, dentro de

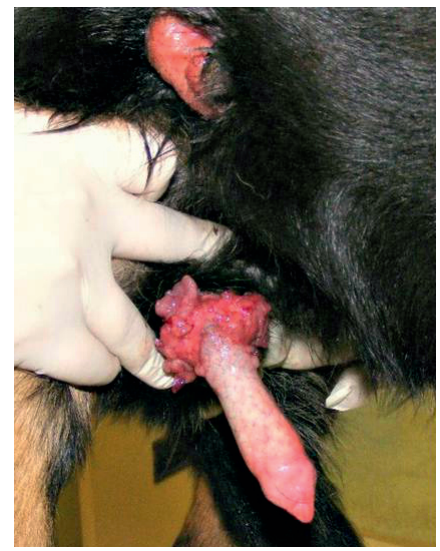

Figura 3. Fotografía de la masa multinodular localizada sobre la mucosa peneana.

Picture of the multinodular mass localized on penis mucosa. la proliferación celular neoplásica se observaron focos de necrosis y restos celulares. La zona marginal se observó con amplias hemorragias difusas. En la periferia de la proliferación celular se observaron restos de tejido conectivo y algunos vasos sanguíneos. Los hallazgos observados fueron compatibles con tumor venéreo transmisible.

Finalmente, pruebas moleculares confirmaron el TVT por medio de LINE-1 (Long Interspersed Nuclear Element) insertado en el oncogen c-myc. Para llevar a cabo la técnica se extrajo el ADN genómico de todas las zonas y se realizó diagnóstico molecular mediante PCR, con el empleo de un par de iniciadores LINEAS-1/ Myc.s. Como resultado se obtuvieron amplicones de 550 pb (figura 4) según lo reportado por Liao y col (2003).

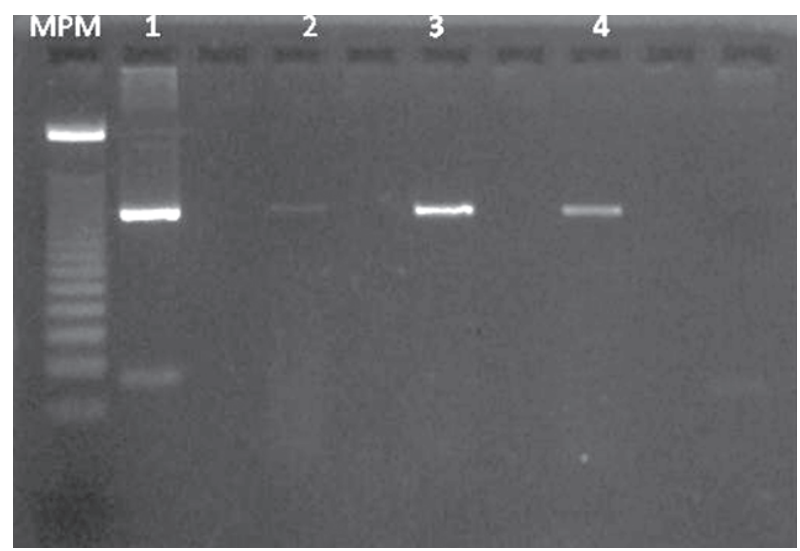

Figura 4. Imagen del PCR realizado a las muestras de TVT obtenidas de pene, párpados y piel. Se observa la inserción de LINE-1 en el oncogen c-myc. Carril 0: marcador de peso molecular (MPM), carril 1; control positivo, carril 2: muestra tejido de pene, carril 3; muestra tejido piel, carril 4; muestra tejido párpado.

PCR reaction in CTVT samples from penis, eyelids and skin. The image shows LINE-1 insertion in oncogene c-myc. Line 0: Molecular weight marker (MPM), line 1: Positive control, line 2: penis, line 3: skin, line 4: eyelids. 


\section{RESULTADOS Y DISCUSIÓN}

\section{TRATAMIENTO Y EVOLUCIÓN}

Establecido el diagnóstico de TVT, se procedió con un protocolo quimioterápico a base de sulfato de vincristina (Pfizer Pty Limited, Australia), 0,5 mg/ $\mathrm{m}^{2} \mathrm{IV}$, diluido en $20 \mathrm{ml}$ de $\mathrm{NaCl}$ 0,9\% con repeticiones cada 7 días. Para disminuir la contaminación de las heridas se trató con amoxicilina/ácido clavulánico (Laboratorio Chile, Chile) $20 \mathrm{mg} / \mathrm{kg}$ cada 12 horas durante 10 días. El paciente quedó hospitalizado para evitar contacto con otros perros y controlar la evolución. Previo a cada sesión de quimioterapia se realizó un hemograma de control.

Con el tratamiento se observó evidente mejoría a partir de la primera semana. Posterior a la tercera administración de vincristina las lesiones genitales desaparecieron. Las lesiones de piel, progresivamente disminuyeron de tamaño, lo mismo que su descarga purulenta. La queratitis bilateral desapareció posterior al segundo tratamiento, siendo asociada las lesiones a un efecto traumático de la masa sobre la córnea. Previo a la sesión 4 se detectó que el hematocrito se encontraba en 24\% (RR 37-50\%) con un recuento de eritrocitos de $3,49 \times 10^{6}$ cels/uL (RR 5.5-8,5 cels/uL), VCM:68 fl (RR 60-77fl), CHbCM:326 g/L (RR 320-370 g/L) e IPR: 1,68, esto último se asoció a una baja regeneración respecto del hematocrito que presentaba. Para evitar agravar la anemia no regenerativa el tratamiento fue suspendido por 15 días, hasta que el hematocrito alcanzó $30 \%$. La terapia con sulfato de vincristina fue efectiva para producir la regresión de todos los tumores en 6 sesiones, sin mostrar recurrencia.

El TVT canino se localiza preferentemente en la zona genital tanto de machos como hembras. La localización extragenital es posible observarla en $23 \%$ de los perros afectados de TVT (Kabuusu y col 2010). En este caso la forma del tumor localizado en la base del pene fue un granuloma firme hiperémico, pedunculado de unos $5 \mathrm{~cm}$ de diámetro. La presencia de esta lesión resultó ser un hallazgo, ya que las lesiones más predominantes se encontraban en la piel, las que se encontraban contaminadas con secreción purulenta, concordante con los resultados observados en el hemograma. Sin embargo, no se pudo concluir si las lesiones cutáneas fueron primarias o secundarias a la lesión genital.

El comportamiento de las metástasis del TVT no está claro. El TVT canino es una neoplasia de carácter benigno con bajas tasas de metástasis. Las metástasis pueden ser generadas de manera autóloga o también de manera heteróloga. La conducta de los perros como lamer, oler y morder, especialmente en los vagos facilita la transmisión y presencia de metástasis (Silva y col 2007). La forma cutánea del TVT, aunque es rara en la práctica, ha sido descrita como lesiones localizadas o generalizadas (Park y col 2006). También se han reportado metástasis intracraneales y en tercer párpado (Ferreira y col 2000) .
El comportamiento biológico del TVT es variable y depende de la respuesta inmune del perro contagiado. Las metástasis del TVT ocurren usualmente en perros con condiciones fisiológicas alteradas como inmunosupresión, malnutrición o en animales jóvenes (Kubuusu y col 2010, Park y col 2006, Ferreira y col 2000). En el presente caso, las múltiples neoplasias extragenitales podrían asociarse a una mala nutrición por la mala condición corporal al momento del diagnóstico. La presencia de TVT cutáneo se asocia al trasplante tipo xenoinjerto por el contacto directo de la piel con el tumor o secreciones con células tumorales. Por otro lado, la habilidad del TVT de generar metástasis hacia órganos internos puede ser vía linfática o hematógena pudiendo aumentar en estados de inmunosupresión (Kubuusu y col 2010).

A pesar de que el CTVT provoca una respuesta inmunológica humoral y celular, las células tumorales atraviesan la barrera del Complejo Mayor de Histocompatibilidad (MHC). La proliferación tumoral en el tejido sano se debe a que la expresión proteica de MHC I y b-globulina es baja (Algarra y col 2004, Cabrera y col 2003, GarcíaLora y col 2003). Incluso puede ocurrir una pérdida total de las moléculas clase I provocada por cambios en la bmicroglobulina mediante mutaciones, alteraciones en el procesamiento del antígeno, pérdida de haplotipo, pérdidas alélicas, downregulation o fenotipos compuestos (Murgia y col 2006). Sin embargo, la concentración de MHC I y b2-microglobulinas llegan a niveles normales durante la regresión tumoral (Murgia y col 2006, Yang y col 1987).

El diagnóstico microscópico del TVT canino, a partir de improntas para citología o histopatología constituyen el procedimiento de elección siendo las características microscópicas concluyentes de diagnóstico (Pigatto y col 2011). En este caso, la presentación de tumores en distintas zonas pudo haber estado asociada al tipo celular encontrado, el que fue clasificado como plasmocítico. Se describen 3 tipos citomorfológicos de TVT, linfocítico, plasmocítico y mixto, que se diferencian en cuanto al comportamiento biológico, especialmente respuesta a la quimioterapia y agresividad (Flórez y col 2012). El plasmocítico se considera el más común tipo morfológico y el que se asocia a presentaciones más agresivas. Por otro lado, no se han detectado diferencias entre tipos morfológicos y su respuesta frente a la quimioterapia con vincristina (Rocha y col 2013). Sin embargo, aparentemente el subtipo plasmocítico del TVT presenta una bomba que permite sacar la vincristina y la doxorrubicina de la célula, lo que reduciría sus concentraciones intracelulares a dosis no letales contribuyendo a una quimiorresistencia (Flórez y col 2012). A pesar de que se ha señalado el transposón LINE-1 insertado en el oncogen c-myc como una alteración molecular específica para TVT (Amariglio y col 1991), polimorfismo genético asociado a diferentes fenotipos no se ha detectado (Fonseca y col 2012). Por otro lado, nuestros hallazgos indican que tampoco hay polimorfismo genético de acuerdo con la ubicación del TVT en el 
perro, ya que la inserción de LINE-1/c-myc se observó en todas las muestras. Como la inserción de LINE-1/c-myc no ocurre en perros sanos, este podría usarse como un marcador tumoral (Fonseca y col 2012).

En el presente reporte el tratamiento con vincristina fue suficiente para eliminar el TVT. Habitualmente se sugieren 4 sesiones cada 7 días para lograr una remisión total (Lorimier y Fan 2007). El régimen de administración de vincristina está ligado al nadir de mielosupresión que es en promedio 5 días. Por lo tanto se sugiere una administración semanal con dosis que pueden fluctuar entre 0,5 y $0,75 \mathrm{mg} / \mathrm{m} 2$ (Lorimier y Fan 2007, Wilson y Barton 2012). En el presente caso se realizaron 6 sesiones en total usando $0,5 \mathrm{mg} / \mathrm{m} 2$. Algunos estudios han demostrado que un promedio de 5 sesiones logra una remisión total del TVT, con un rango de 1-16 sesiones (Scarpelli y col 2010). La disminución del hematocrito se consideró un efecto secundario de la aplicación de vincristina, a pesar de que los valores de los neutrófilos se mantenían dentro del rango de normalidad. Sin embargo, la anemia asociada a quimioterapéuticos es rara debido a la larga vida media de los eritrocitos (120 días), aun cuando esta se caracteriza por ser leve y paulatina (Larson 2009, MacDonald 2009). En humanos, la presencia de anemia durante quimioterapia puede estar asociada a hemorragias gástricas, insuficiencia renal o causas nutricionales. Lo importante es definir la causa, ya que los tratamientos difieren considerablemente (Steensma 2008). En este caso la suspensión de la terapia y un aumento en la condición corporal durante la pausa del tratamiento pudieron ser necesarios para recuperar los niveles normales de eritrocitos.

A pesar de la presentación y diseminación poco común del CTVT, la monoterapia con vincristina fue efectiva para remitir las lesiones. Sin embargo, no se pudo determinar el origen primario del tumor pudiendo asumir que la diseminación del TVT se relacionó con la caracterización celular de tipo plasmocítica.

\section{REFERENCIAS}

Algarra I, A Garcia-Lora, T Cabrera, F Ruiz-Cabello, F Garrido. 2004. The selection of tumor variants with altered expression of classical and nonclassical MHC Class I molecules: implications for tumor immune escape. Cancer Immunol Immunother 53,904-910.

Amariglio E, I Hakim, F Brok-simoni, Z Grossman, N Katzir, A Harmelint, B Ramot, G Rechavi. 1991. Identity of rearranged LINE/c-MYC junction sequences specific for the canine transmissible venereal tumor. PNAS 88, 8136-8139.

Cabrera T, MA Lopez-Nevot, JJ Gaforio, F Ruiz-Cabello, F Garrido.2003. Analysis of HLA expression in human tumor tissues. Cancer Immunol Immunother 52, 1-9.

Ferreira AJ, A Jaggy, AP Varejao, ML Ferreira, JM Correia, JM Mulas, O Almeida, P Oliveira, J Prada. 2000. Brain and ocular metastases from a transmissible venereal tumour in a dog. J Small Anim Pract 41, 165-168.

Flórez MM, F Pedraza, F Grandi, NS Rocha. 2012. Cytologic subtypes of canine transmissible venereal tumor. Vet Clin Pathol 41, 4-5.
Fonseca L, L Mota, M Colodel, I Ferreira, C Brandao, N Rocha. 2012. Spontaneous canine transmissible venereal tumor: association between different phenotypes and the insertion LINE-1/c-myc. Rev Colomb Cienc Pecu 25, 402-408.

García-Lora A, I Algarra, F Garrido. 2003. MHC class I antigens, immune surveillance, and tumor immune escape. J Cell Physiol 195, 346-355.

Grandez R, CM de Priego, P Yi, L Torres. 2011. Tumor venéreo transmisible canino extragenital: Estudio retrospectivo de 11 casos. Rev Inv Vet Perú 22, 342-350.

Kabuusu RM, DF Stroup, C Fernandez. 2010. Risk factors and characteristics of canine transmissible venereal tumours in Grenada, West Indies. Vet Comp Oncol 8, 50-55.

Kangasniemi M, RJ McNichols, JA Bankson, A Gowda, RE Price, JD Hazle. 2004. Thermal therapy of canine cerebral tumors using a 980 $\mathrm{nm}$ diode laser with MR temperature-sensitive imaging feedback. Lasers Surg Med 35, 41-50.

Larson V. 2009. Complications of Chemotherapeutics Agents. In: Silverstein D, Hopper K (eds). Small Animal Critical Care Medicine. Saunders Elsevier, St Louis, Mo, USA, Pp 817-820.

Levy E, ME Mylonakis, MN Saridomichelakis, ZS Polizopoulou, V Psychogios, AF Koutinas. 2006. Nasal and oral masses in a dog. Vet Clin Pathol 35, 115-118.

Liao K, Z Lin, H Pao, S Kam, F Wang, R Chu. 2003. Identification of canine transmissible venereal tumor cells using in situ polymerase chain reaction and the stable sequence of the long interspersed nuclear element. J Vet Diagn Invest 15, 399-406.

Lorimier de L, T Fan. 2007. Canine transmissible vereneal tumor. In: Withrow S, Vail D (eds). Small animal clinical oncology. Saunders Elsevier, Philadelphia, USA, Pp 799-804.

MacDonald V. 2009. Chemotherapy: managing side effects and safe handling. Can Vet J 50, 665-669.

Marcos R, M Santos, C Marrinhas, E Rocha. 2006. Cutaneous transmissible venereal tumor without genital involvement in a prepubertal female dog. Vet Clin Pathol 35, 106-109.

Murgia C, JK Pritchard, SY Kim, A Fassati, RA Weiss. 2006. Clonal origin and evolution of a transmissible cancer. Cell 126, 477-487.

Ortega-Pacheco A, M Acevedo-Arcique, CH Sauri-Arceo, M BolioGonzález, E Gutiérrez-Blanco. 2003. Prevalência de tumor venéreo transmissible em perros callejeros de la ciudad de Mérida, Yucatán, México. Rev Biomed 14, 83-87.

Park MS, Y Kim, MS Kang, SY Oh, DY Cho, NS Shin, DY Kim. 2006. Disseminated transmissible venereal tumor in a dog. $J$ Vet Diagn Invest 18, 130-133.

Pigatto JATP, PS Hünning, BS Bercht, L de Albuquerque. 2011. Transmissible venereal tumor in the palpebral conjunctiva of a dog: case report. Semina 32, 1139-1144.

Scarpelli C, M Valladão, K Metze. 2010. Predictive factors for the regression of canine transmissible venereal tumor during vincristine therapy. Vet J 183, 362-363.

Silva MCV, RR Barbosa, RC dos Santos, RSN Chagas, WP Costa. 2007. Avaliação epidemiológica, diagnóstica e terapêutica do tumor venéreo transmissível (TVT) na população canina atendida no hospital veterinário da UFERSA. Acta Vet Brasílica 1, 28-32.

Steensma D. 2008. Is Anemia of Cancer Different From Chemotherapy Induced Anemia?. J Clin Oncol 26, 1022-1024.

Rocha C, R Rabelo, V Vulcani, A Pereira, P Almeida, L Batista, V Brianezi. 2013. Morphological patterns and malignancy criteria of transmissible venereal tumor in cytopathological and histopathological exams. Braz J Vet Res Anim Sci 50, 238-246.

Yang TJ, JP Chandler, S Dunne-Anway.1987. Growth stage dependent expression of MHC antigens on the canine transmissible venereal sarcoma. Br J Cancer 55,131-134.

Wilson H, C Barton. 2012. Chemotherapy. In Boothe DM (ed). Small Animal Clinical Pharmacology and Therapeutics. $2^{\text {nd }}$ ed. Elsevier Saunders, St. Louis, Missouri, USA, Pp 1210-1235. 
